\title{
Bariatric surgery in patients with Type 2 diabetes: benefits, risks, indications and perspectives
}

\author{
A.-J. Scheen ${ }^{\mathrm{a}}$, J. De Flines ${ }^{\mathrm{a}}$, A. De Roover ${ }^{\mathrm{b}}$, N. Paquot ${ }^{\mathrm{a}}$ \\ ${ }^{a}$ Division of Diabetes, Nutrition and Metabolic Disorders, Department of Medicine, CHU Sart Tilman, University of Liège, Liège, Belgium. \\ ${ }^{b}$ Division of Gastrointestinal Surgery, Department of Surgery, CHU Sart Tilman, Liège, Belgium.
}

\begin{abstract}
Obesity plays a key role in the pathophysiology of type 2 diabetes (T2DM), and weight loss is a major objective, although difficult to achieve with medical treatments. Bariatric surgery has proven its efficacy in obtaining marked and sustained weight loss, and is also associated with a significant improvement in glucose control and even diabetes remission. Roux-en-Y gastric bypass appears to be more effective in diabetic patients than the restrictive gastroplasty procedure. This may be explained not only by greater weight reduction, but also by specific hormonal changes. Indeed, increased levels of glucagon-like peptide-1 (GLP-1) and glucose-dependent insulinotropic polypeptide (GIP) may lead to improved $\beta$-cell function and insulin secretion as well as reduced insulin resistance associated with weight loss. The presence of T2DM in obese individuals is a further argument to propose bariatric surgery, and even more so when diabetes is difficult to manage by medical means and other weight-related complications are present. Bariatric surgery is associated with a better cardiovascular prognosis and reduced mortality, even though acute and long-term complications may occur. The observation that surgical rerouting of nutrients triggers changes in the release of incretin hormones that, in turn, ameliorate the diabetic state in the absence of weight loss has led to the recent development of innovative surgical procedures. Thus, bariatric surgery may be said to be progressing towards so-called 'metabolic surgery', which merits further evaluation in patients with T2DM within a multidisciplinary approach that involves both surgeons and endocrinologists.
\end{abstract}

Keywords : Bariatric surgery ; Gastric bypass ; Gastroplasty ; Obesity ; Type 2 diabetes ; Review

\section{Résumé}

\section{Chirurgie bariatrique chez les patients atteints de diabète de type 2: bénéfices, risques, indications et perspectives}

L'obésité joue un rôle-clé dans la physiopathologie du diabète de type 2 (DT2) et l'obtention d'une perte de poids est un objectif majeur, bien que difficile à atteindre avec les moyens médicaux. La chirurgie bariatrique a apporté la preuve de son efficacité pour induire un amaigrissement marqué et soutenu, qui s'accompagne d'une amélioration du contrôle glycémique et assez souvent d'une remission du diabète. La dérivation gastrique avec l'anse de Roux-en-Y apparaît être plus efficace chez les patients diabétiques que la simple technique restrictive qu'est la gastroplastie calibrée. Ceci peut s'expliquer non seulement par une perte pondérale supérieure, mais aussi par des modifications hormonales spécifiques. En effet, une augmentation des concentrations de GLP-1 (glucagon-like peptide-1) et de GIP (glucose-dependent insulinotropic polypeptide) avec la dérivation permet d'améliorer la fonction de la cellule $\beta$ et la réponse insulinosécrétoire, en plus de la diminution de l'insulinorésistance consécutive à l'amaigrissement. La présence d'un DT2 chez un sujet obèse est un argument supplémentaire pour proposer une chirurgie bariatrique, d'autant plus que le diabète est difficile à gérer avec les moyens médicaux et qu'il existe d'autres complications liées à l'excès de poids. La chirurgie bariatrique est associée à un meilleur pronostic cardiovasculaire et à une diminution de la mortalité, même si cette chirurgie peut également occasionner des complications à court et à long terme. L'observation que la dérivation gastrointestinale des aliments stimule la sécrétion des hormones incrétines, susceptibles d'améliorer le diabète en l'absence de perte de poids, a conduit récemment au développement de nouvelles procédures chirurgicales. Ainsi, la chirurgie bariatrique pourrait évoluer vers une chirurgie métabolique. Cette dernière mérite d'être mieux évaluée chez les patients DT2, dans une approche multidisciplinaire impliquant une collaboration étroite entre chirurgiens et endocrinologues.

Mots clés : Chirurgie bariatrique ; Dérivation gastrique ; Diabète de type 2 ; Gastroplastie ; Obésité ; Revue 


\section{Introduction}

Obesity is a major independent risk factor for the development of type 2 diabetes mellitus (T2DM) and is also associated with the rapidly increasing prevalence of diabetes $[1,2]$. The majority of patients $(>80 \%)$ diagnosed with T2DM are overweight [body mass index $(\mathrm{BMI})>25 \mathrm{~kg} / \mathrm{m}^{2}$ ], and roughly $50 \%$ are obese $\left(\mathrm{BMI}>30 \mathrm{~kg} / \mathrm{m}^{2}\right)$ and almost $10 \%$ are morbidly obese (BMI $\left.>40 \mathrm{~kg} / \mathrm{m}^{2}\right)$. This twin epidemic of obesity and diabetes has serious consequences with increased cardiovascular morbidity and premature mortality. However, studies have shown that weight loss, even when modest, can reduce the incidence of T2DM in patients with impaired glucose tolerance and improve blood glucose control (and other cardiovascular risk factors) in patients with T2DM, while marked weight loss can even lead to resolution/remission of diabetes [2].

Lifestyle interventional programmes including diet therapy, behavioural modification, exercise regimens and pharmacotherapy are widely used in various combinations $[3,4]$. Unfortunately, clinically significant weight loss is uncommon and mostly transient, particularly in patients with severe obesity and with T2DM, for whom sustained weight reduction is even more difficult to achieve.

Bariatric surgery is a rapidly evolving branch of surgical science [5-7]. The aim is to induce major weight loss in those whose obesity places them at high risk of severe health problems, including T2DM [7-9]. In an attempt to balance the risks of surgery against the benefits of weight loss, bariatric operations are currently performed only in the morbidly obese (BMI $>40 \mathrm{~kg} / \mathrm{m}^{2}$ ) and in those with a BMI $>35 \mathrm{~kg} / \mathrm{m}^{2}$ and co-morbidities such as T2DM [10]. In the mid-1990s, Pories et al. [11] proposed the provocative theory that T2DM might be a surgical disease, as bariatric surgery proved to be the most effective approach for treating and even resolving this type of diabetes [12]. Over the past 10 years, bariatric surgery has gained an increasing place in the management of obese patients with T2DM [13-16]. Moreover, the suggestion that the foregut plays an important role in the pathophysiology of T2DM opens up new possibilities for a surgical approach in patients with T2DM, even in absence of severe obesity, as well as a nominal shift from 'bariatric'to 'metabolic'surgery $[17,18]$.

The present review focuses mainly on the benefit- risk profile of bariatric surgery in the management of obese patients with T2DM, but also includes a discussion of the mechanisms of action of various surgical procedures, the factors influencing prognosis and validated indications in clinical practice. In addition, new perspectives for metabolic surgery in non-obese patients with T2DM are also briefly discussed.

\section{Surgical techniques}

A number of surgical approaches to induce weight loss have been developed, and several are also currently used in the management of obese patients with T2DM [5, 6]. In general, these procedures can be classified as: solely restrictive [laparoscopic adjustable gastric banding (LAGB) and its variant, vertical banded gastroplasty (VGB)] [19]; mostly restrictive [Roux-en-Y gastric bypass (RYGB)] [20]; and mostly malabsorptive [biliopancreatic diversion with duodenal switch (BPDS)] [21]. In the first group, the mechanism essentially hinges upon generating effective satiety signals with only small amounts of ingested food [19]. In the second group, a degree of gastric restriction is coupled with bypass of the duodenum and upper jejunum, a procedure that may also result in important hormonal changes for glycaemic control (see below) [20]. In the third group of procedures, only the final $50 \mathrm{~cm}$ of the total length of the bowel is available for ingested food and biliopancreatic juices to mix, leading to consistent nutrient malabsorption [21].

These techniques have all been proven effective in the management of obese patients with or without T2DM. However, as these surgical procedures are different, their efficacy-to-safety balance may also differ. Techniques with a component of malabsorption generally lead to more pronounced and more sustained weight loss compared with solely restrictive procedures [22]. As for tolerability and safety, patients treated with LAGB had lower short-term morbidity than those treated with RYGB, but repeat-operation rates are higher among patients who undergo LAGB. However, according to a recent Cochrane review, although certain procedures result in greater weight loss, well-validated comparative data are nevertheless limited. The evidence for safety is even less clear. Because of the limited evidence and poor quality of trials, the reviewers concluded that caution is required when interpreting comparative safety and effectiveness [23].

Innovative bariatric surgical procedures continue to be under investigation. The gastric sleeve, or sleeve gastrectomy, is certainly among the more advanced and already in routine use in some centres [24,25]. The procedure results in a narrow gastric tube through excision of most of the stomach. The operation does more than just limit intake, but also removes most — or perhaps all—of the ghrelin-producing cells in the gastric mucosa. In addition, two new procedures - the duodenojejunal bypass stomach-sparing operation [26] and ileal transposition 
[27-29] - have been tested in attempts to induce remission of diabetes without weight loss in lean or only modestly overweight patients with T2DM. However, both are still in the early stages of human trials (see Perspectives below). Nevertheless, there is little doubt that the mechanism of action on glucose metabolism differs depending on the type of bariatric surgery [30].

\section{Mechanisms of metabolic improvement}

Bariatric surgery is a highly effective means of inducing diabetes remission in very obese patients with T2DM [31]. Diabetes remission results from improvements in both insulin resistance and $\beta$-cell dysfunction. Better insulin action on glucose metabolism relieves secretory pressure on the $\beta$-cell, resulting in reduced insulin output $[32,33]$. However, significant improvements in dynamic $\beta$-cell responses may also play a role. This may be the result of reduced glucotoxicity and/or specific incretin effects favouring $\beta$-cell function. By rank order of increasing efficacy in glucose control, the most common surgical procedures go from the solely restrictive to the mostly restrictive and to the mostly malabsorptive, thus paralleling their weight-reducing effects. The mechanisms responsible for glycaemic improvement and resolution/ remission of diabetes after bariatric surgery also depend on the type of surgical procedure used.

Calorie restriction and weight loss are the dominant mechanisms of improved glucose metabolism when solely restrictive procedures are applied $[8,19]$. The former appears to account for the early post-surgical recovery of insulin sensitivity and secretory dynamics, while the latter is the final determinant of outcome once weight and caloric balance have stabilized [32, 33]. In general, when analyzing the effect of solely restrictive procedures, the sustained improvement of glucose control is directly proportional to the final amount of weight loss. However, even with RYGB, the percentage of weight lost is a predictive factor of diabetes remission [34].

When food transit is surgically altered, changes in the pattern of gastrointestinal hormone release may support early adaptation of $\beta$-cell function, but this is unlikely to make a major contribution to insulin action. Weightindependent antidiabetic effects with RYGB are evident from the rapid resolution of T2DM (before weight loss occurs), the greater improvement of glucose homoeostasis after RYGB than after equivalent weight loss by other means and the occasional development of very-late-onset pancreatic $\beta$-cell hyperfunctioning. Several mechanisms probably mediate the direct antidiabetic impact of RYGB, including: enhanced nutrient stimulation of L-cell peptides [for example, glucagon-like peptide-1 (GLP-1)] from the lower intestine ('hindgut hypothesis'); the intriguing, but as yet uncharacterized, phenomena related to exclusion of the upper intestine from contact with ingested nutrients ('foregut hypothesis'); compromised ghrelin secretion; and most likely other effects that have yet to be identified $[30,35,36]$. Indeed, a role for glucagon or gut-derived glucagonotropic signalling as putative diabetogenic signals from the foregut has been recently proposed [37]. Research designed to prioritize these mechanisms and identify potential additional mechanisms promises to help in the optimalization of surgical design (see Perspectives below) and may also reveal novel pharmaceutical targets for antidiabetes drug treatments [36].

\section{Clinical benefits}

The dataset of a recent systematic review and meta-analysis included 621 studies with 888 treatment arms and 135,246 patients; of these studies, 103 treatment arms, involving 3188 patients, reported resolution of diabetesthat is, resolution of the clinical and laboratory manifestations of T2DM [38]. In addition, 19 studies with 43 treatment arms and 11,175 patients reported both weight loss and diabetes resolution in 4,070 diabetic patients. At baseline, the patients'mean age was 40.2 years, BMI was $47.9 \mathrm{~kg} / \mathrm{m}^{2}, 80 \%$ were female and $10.5 \%$ had undergone previous bariatric procedures. Meta-analysis showed an overall loss of $38.5 \mathrm{~kg}$ or $56 \%$ of excess body weight. Also, $78 \%$ of the diabetic patients enjoyed complete resolution of their disease, while diabetes was improved or resolved in nearly $87 \%$. Weight loss and diabetes resolution were greatest for patients undergoing BPDS, followed by RYGB, and was lowest with LAGB. Insulin levels declined significantly postoperatively, as did glycated haemoglobin $\left(\mathrm{HbA}_{1 \mathrm{c}}\right)$ and fasting glucose values. Weight and diabetes parameters showed little differences within 2 years, or after 2 or more years, of follow-up. The conclusion was that the clinical and laboratory manifestations of T2DM were resolved or improved in the vast majority of patients after bariatric surgery, and these responses are more pronounced with procedures that led to a greater percentage of excess weight loss maintained for 2 or more years.

Besides the effect on glucose control, bariatric surgery also leads to significant improvement of cardiovascular risk factors, especially those linked with the metabolic syndrome, including inflammation markers [39]. The prospective Swedish Obese Subjects (SOS) study confirmed that bariatric surgery significantly improves glucose, lipid and blood pressure control in surgically compared with medically treated obese individuals [40]. 
Recently, the 10.9-year follow-up of the SOS study reported a significant 30\% risk reduction in overall mortality in 2,010 obese patients (7.4\% with T2DM) who had undergone bariatric surgery [41]. Likewise, in a retrospective US cohort of 7,925 surgical patients mostly treated with RYGB, mortality from any cause was significantly $40 \%$ lower than in 7,925 non-surgical obese patients [42]. However, mortality data for a specific diabetic cohort are scanty. In an early retrospective analysis of two groups of obese diabetic patients, the mortality rate (including perioperative deaths) in the control medical group was $28 \%$ after 6.2 years compared with only $9 \%$ in the RYGB surgical group after 9 years. For every year of follow-up, patients in the control group had a $4.5 \%$ risk of dying vs $1.0 \%$ for those in the surgical group. The improvement in mortality with surgery was primarily due to a decrease in the number of cardiovascular deaths [43].

Recently, a systematic review concluded that bariatric surgery appears to be a clinically and cost-effective intervention for moderate-to-severely obese people compared with non-surgical interventions [44]. However, uncertainties persist and further research is required. In particular, new research needs to investigate the resolution and/or development of T2DM and, more important, the duration of T2DM remission, so that the potential benefits of early intervention may be better assessed.

\section{Prognostic factors}

Studies of postoperative outcomes for bariatric surgery have provided information on the predictors of success. Indeed, the surgeon's and institution's experience, and patients'behaviour after surgery, are key determinants of its success or failure in all obese subjects, including those with T2DM [45].

Rates of total diabetes remission and glycaemic improvement essentially depend on the type of surgical intervention, as already stated. Although physiological (hormonal) mechanisms probably contribute to RYGB outcomes, early rapid weight loss and the percentage of excess weight lost were also significant factors associated with diabetes remission in a multivariate analysis [34]. In addition, in that study, the preoperative insulin dose was another predictor, suggesting that the severity of diabetes plays a crucial role. This may be indexed by disease duration, $\mathrm{HbA}_{1 \mathrm{c}}$ level, intensity of treatment and presence of complications [46]. Diabetes duration is generally related to a progressive reduction in $\beta$-cell function and/or mass, which may hinder remission of diabetes even after marked weight loss if surgery is performed too late. $\mathrm{HbA}_{\mathrm{lc}}$ is a classical marker not only of glucose control, but also, indirectly, of diabetes severity. The higher the initial $\mathrm{HbA}_{1 \mathrm{c}}$ level, the lower the chances of diabetes remission after bariatric surgery. Similarly, the intensity of treatment also plays a major part. Insulin-treated patients are less prone to complete remission of diabetes compared with patients taking oral treatment, and recovery of adequate glucose control despite stopping insulin therapy would be an alternativeand perhaps more realistic - efficacy criterion in such a diabetic subgroup. Among patients using oral therapy, the rate of complete diabetes remission is higher in those treated with monotherapy compared with those already receiving the maximum oral combined treatment. The presence of diabetic complications (such as nephropathy and retinopathy) should probably be considered an indirect marker of longstanding, poorly controlled diabetes rather than a direct contributor to surgical treatment failure.

Other factors, such as adiposity topography, T2DM family history, interaction with previous antidiabetic therapies and evidence of autoimmunity (late-onset type 1 diabetes) have not been specifically analyzed and merit further evaluation.

\section{Risks}

The risk and type of complications related to bariatric surgery among diabetic patients are not much different from those in a non-diabetic population, although some (such as infections) are more prevalent in diabetics. Surprisingly, bariatric surgery is remarkably safe, especially given the large body size of patients, and the frequency and seriousness of co-morbidities. Nevertheless, the operative mortality rate is low: the 30-day mortality rates reported in a recent meta-analysis were $\mathrm{LAGB}=0.1 \%, \mathrm{VBG}=0.1 \%, \mathrm{RYGB}=0.5 \%$ and $\mathrm{BPDS}=$ $1.1 \%$ [47]. However, death may also occur after discharge from hospital, possibly due to pulmonary embolism and arrhythmias [6].

Surgical complications are either acute or long term [6]. Acute complications occur in 5-10\% of patientsdepending on the procedure, and the patients'risk, age and condition - and mirror those following other abdominal operations, including haemorrhage, obstruction, anastomotic leaks, infection, arrhythmias and pulmonary emboli. Long-term complications are miscellaneous and mostly include neuropathies due to nutritional deficiencies, internal hernias, anastomotic stenoses and emotional disorders. Although nutritional deficits can be avoided with daily multivitamin and mineral supplements, compliance with this recommendation 
is not universal, and dramatic complications due to severe deficiencies may occur, albeit rarely. In general, however, the complications do not differ in diabetic, compared with non-diabetic, patients [48].

The risk of hypoglycaemia sometimes reported in non-diabetic individuals late after RYGB (attributed to the development of neisidioblastosis) does not appear to affect diabetic patients. However, calorie restriction, weight loss and incretin-related mechanisms may dramatically improve glucose control and lead to early hypoglycaemia if no appropriate reduction in glucose-lowering therapies is made soon after surgery. Ideally, this should be performed as a preventative approach rather than waiting for hypoglycaemic episodes to occur before considering adjustment of any antidiabetic treatment [7]. Similarly, antihypertensive therapy, commonly used in obese patients with T2DM, should be frequently adjusted after bariatric surgery to avoid hypotension leading to orthostatic dizziness.

\section{Indications}

The first indications and contraindications for bariatric surgery were established in 1991 by the US National Institutes of Health (NIH) Consensus Conference on surgery for obesity [10]. In 2004, the American Society for Bariatric Surgery (ASBS; now the American Society for Metabolic and Bariatric Surgery, or ASMBS), updated that statement with a follow-up Consensus Conference [49]. In practical terms, most physicians, surgeons and carers consider patients eligible for bariatric surgery if their BMI is at least $40 \mathrm{~kg} / \mathrm{m}^{2}$, or at least $35 \mathrm{~kg} / \mathrm{m}^{2}$ if accompanied by co-morbidities such as T2DM, hypertension, severe arthritis (limiting daily function) and cardiopulmonary failure. In the past, the age limit was 18-65 years, but recent data show that teenagers and patients over 65 can benefit from surgery with little or no increases in risk. Other inclusion criteria include the patient's ability to understand the surgery and its consequences, and to comply with long-term follow-up, and the patient's agreement to maintain vitamin and mineral supplementation, and to report problems promptly to specialists familiar with the complications of bariatric surgery. Similar recommendations were made in the 2007 Interdisciplinary European guidelines for surgery for those with severe (morbid) obesity [50].

Thus, T2DM as a complication of obesity is a further argument to propose bariatric surgery even when BMI is $35-40 \mathrm{~kg} / \mathrm{m}^{2}$, as stated in the official recommendations [10, 49, 50]. From a practical point of view, it is reasonable to consider as major indications either those patients whose T2DM is difficult to manage or poorly controlled mainly because of the presence of severe obesity, or those who, in addition to diabetes, have several risk factors directly related to excess weight that may markedly worsen their overall prognosis (such as hypertension, dyslipidaemia and sleep apnoea). However, the choice of procedure is not yet based on sound data [23], although many surgeons currently favour either RYGB or the duodenal switch over LAGB in patients with T2DM for reasons discussed above [7, 15, 16, 22].

\section{Perspectives}

Certain types of bariatric surgical procedures have proved not only to be effective for treating obesity, but also appear to be associated with endocrine changes that, independent of weight loss, give rise to remission of T2DM $[37,51]$. Observations in animals and in humans suggest that changes in the gut hormonal milieu after bypass of the distal stomach, duodenum and proximal jejunum can influence T2DM, despite the absence of significant weight loss $[17,51]$. As a result, the use of bypass bariatric surgery and experimental gastrointestinal manipulations to treat T2DM is increasing among less obese patients (BMI $<35 \mathrm{~kg} / \mathrm{m}^{2}$ or even $<30 \mathrm{~kg} / \mathrm{m}^{2}$ ). While BMI currently represents a significant indication for bariatric surgery (see Indications above), evidence shows that, so far, no clear cut-off BMI score accurately predicts successful surgical outcomes. Furthermore, BMI appears to have limited value in defining the risk profile in T2DM patients. For this reason, the current BMI-based criteria for bariatric surgery may not be an adequate indication for such patients [17].

There is also increasing evidence that bariatric operations may exert intrinsic antidiabetic actions beyond weight loss. Malabsorptive operations currently offer the best chances of revealing weight-independent mechanisms of diabetes resolution, but other smart manipulations of food passage may open up entirely new avenues of treatment. At present, it is speculated that surgical rerouting of nutrients triggers changes in the release of gastrointestine-derived hormones that, in turn, ameliorate the diabetic state. The hindgut hypothesis states that surgical rerouting of nutrients to the distal part of the small intestine results in increased secretion, and concomitant glucose-lowering effects, of GLP-1, whereas the foregut hypothesis emphasizes that surgical bypass of the foregut prevents the release of hitherto unidentified nutrient-induced diabetogenic signals (? glucagon/glucagonotropic signalling) in susceptible individuals [37]. Recent animal investigations using duodenojejunal bypass, a stomach-preserving experimental model of RYGB, have shown that diabetes control is not a mere collateral effect of the treatment of obesity, but directly results from exclusion of the duodenum and 
proximal jejunum from the flow of nutrients. A recent report has described lean T2DM patients who experienced marked improvement of their diabetes with duodenojejunal exclusion surgery that was independent of changes in BMI, fat distribution and body composition [26]. Such a surgical approach was superior to standard care in achieving better glycaemic control, along with reduction or even interruption in insulin requirements. Also, as an alternative approach, ileal transposition may result in significant endocrine changes in the gut, particularly by promoting secretion of GLP-1, an ileal-produced hormone with a well-known role in T2DM [27]. Promising results with ileal interposition (the so-called 'neuroendocrine break') combined with sleeve gastrectomy were recently reported in a small series of patients with T2DM and no obesity [28, 29]. Adequate glycaemic control was obtained in $>90 \%$ of the patients and several hormonal changes were also observed, especially a marked increase in GLP-1 and GIP (glucose-dependent insulinotropic polypeptide).

Large randomized clinical trials against the best medical care should be prioritized to define the role of bariatric surgery in the management of diabetes. Experiments involving less obese or non-obese diabetic patients, or minimizing weight loss, may provide further evidence in favour of so-called metabolic surgery [18]. However, difficult as they may be to carry out, controlled clinical studies using state-of-the-art methodology (including randomization if possible) are necessary to establish the value of metabolic surgery. It would also be of interest to assess the efficacy/safety of bariatric/metabolic surgery in special populations presenting with diabetes, such as pregnant women, adolescents and individuals who are at least 65 years old [48].

\section{Conclusion}

Numerous studies demonstrate that bariatric surgery for obesity can lead to substantial and sustained weight loss. In addition, patients with T2DM have experienced remission of hyperglycaemia or a reduced need for medication. These are partly explained by weight loss, but also by specific hormonal changes, especially after bypass procedures that alter the passage of food in the gut. This may explain why RYGB appears to be superior to LAGB in obese patients with T2DM. Surgical intervention for patients with more recent diabetes onset may see higher rates of resolution than patients with longer T2DM duration because of less-advanced disease and a better insulin secretory reserve. In addition to better glucose control, dyslipidaemia, hypertension and other risk factors improve more markedly in patients treated by surgery compared with those receiving the optimal medical treatment. Such pleiotropic effects may offer special benefits to those with T2DM, who are also known to present with high cardiovascular risks. Observational studies and the prospective SOS trial suggest that longterm survival is favourable for obese (diabetic) patients following bariatric surgery. This suggests that early surgical intervention in cases of T2DM associated with other weight-related complications may be clinically appropriate in patients for whom operative risks are acceptable, with no contraindications. Finally, new surgical procedures are currently undergoing evaluation and may be proposed in future for management of T2DM, even in the absence of severe obesity. Thus, the old paradigm of bariatric surgery may progress towards a new paradigm of 'metabolic obesity'. Recognizing the need to work as a team across disciplines - in particular, endocrinologists and surgeons - is the first step towards addressing the issues and opportunities that surgery has to offer diabetes care and research.

\section{Conflicts of interest}

The authors have reported no conflicts of interest.

\section{References}

[1] ScheenAJ. Obesity and diabetes. In: The management of obesity and related disorders (Kopelman PG, Ed.), Martin Dunitz Ltd, London, UK, 2001:11-44.

[2] Maggio CA, Pi-Sunyer FX. Obesity and type 2 diabetes. Endocrinol Metab Clin North Am 2003;32:805-22.

[3] Scheen AJ, Lefèbvre PJ. Management of the obese diabetic patient. Diabetes Rev 1999;7:77-93.

[4] Scheen AJ. Current management of coexisting obesity and type 2 diabetes. Drugs 2003;63:1165-84.

[5] Crookes PF: Surgical treatment of morbid obesity. Annu Rev Med 2006;57:243-64.

[6] Pories WJ. Bariatric surgery: risks and rewards. J Clin Endocrinol Metab 2008;93(Suppl 1):S89-96.

[7] Scheen AJ. Place de la chirurgie bariatrique dans le traitement de l'obésité. In: Traité de Diabétologie, 2e édition (Ed: Grimaldi A.), Médecine-Sciences Flammarion, Paris, France, 2009, 387-94. 
Published in : Diabetes \& Metabolism (2009), vol. 35

Status : Postprint (Author's version)

[8] Scheen AJ. Aggressive weight reduction treatment in the management of type 2 diabetes. Diab Metab 1998;23:116-23.

[9] Levy P, Fried M, Santini F, Finer N. The comparative effects of bariatric surgery on weight and type 2 diabetes. Obes Surg 2007; $17: 1248-56$.

[10] National Institutes of Health Consensus Development Panel. Gastrointestinal surgery for severe obesity. Consensus Development Conference Statement. Ann Intern Med 1991;115:956-61.

[11] Pories WJ, MacDonald KG, Flickinger EG, Dohm GL, Sinha MK, Barakat HA, et al. Is type II diabetes mellitus (NIDDM) a surgical disease? Ann Surg 1992;215:633-43.

[12] Pories WJ, Swanson MS, MacDonald KG, Long SB, Morris PG, Brown BM, et al. Who would have thought it? An operation proves to be the most effective therapy for adult-onset diabetes mellitus. Ann Surg 1995;222:339-52.

[13] Pinkey J, Kerrigan D. Current status of bariatric surgery in the treatment of type 2 diabetes. Obes Rev 2004;5:69-78.

[14] Schernthaner G, Morton JM. Bariatric surgery in patients with morbid obesity and type 2 diabetes. Diabetes Care 2008;31(Suppl 2):S297-302

[15] Dixon JB. Obesity and diabetes: The impact of bariatric surgery on type-2 diabetes. World J Surg 2009;33:2014-21.

[16] Vetter ML, Cardillo S, Rickels MR, Iqbal N. Narrative review: effect of bariatric surgery on type 2 diabetes mellitus. Ann Int Med 2009;150:94-103.

[17] Rubino F. Bariatric surgery: effects on glucose homeostasis. Curr Opin Clin Nutr Metab Care 2006;9:497-507.

[18] Schulman AP, Del Genio F, Sinha N, Rubino F. "Metabolic"surgery for the treatment of type 2 diabetes. Endocr Pract 2009;15:624-31

[19] Brancatisano A, Wahlroos S, Matthews S, Brancatisano R. Gastric banding for the treatment of type 2 diabetes mellitus in morbidly obese. Surg Obes Relat Dis 2008;4:423-9.

[20] Schauer PR, Burguera B, Ikramuddin S, Cottam D, Gourash W, Hamad G, et al. Effect of laparoscopic Roux-en Y gastric bypass on type 2 diabetes mellitus. Ann Surg 2003;238:467-85.

[21] Scopinaro N, Papadia F, Camerini G, Marinari G, Civalleri D, Gian Franco A. A comparison of a personal series of biliopancreatic diversion and literature data on gastric bypass help to explain the mechanisms of resolution of type 2 diabetes by the two operations. Obes Surg 2008;18:1035-8.

[22] Tice JA, Karliner L, Walsh J, Petersen AJ, Feldman MD. Gastric banding or bypass? A systematic review comparing the two most popular bariatric procedures. Am J Med 2009;121:885-93

[23] Colquitt JL, Picot J, Loveman E, Cleqq AJ. Surgery for obesity. Cochrane Database Syst Rev 2009;2: CD003641.

[24] Rosenthal R, Li X, Samuel S, Martinez P, Zheng C. Effect of sleeve gastrectomy on patients with diabetes mellitus. Surg Obes Relat Dis $2009 ; 5: 429-34$

[25] Vidal J, Ibarzabal A, Romero F, Delgado S, Momblan D, Flores L, Lacy A. Type 2 diabetes mellitus and the metabolic syndrome following sleeve gastrectomy in severely obese subjects. Obes Surg 2008;18:1077-82.

[26] Geloneze B, Geloneze SR, Fiori C, Stabe C, Tambascia MA, ChaimEA, et al. Surgery for nonobese type 2 diabetic patients: an interventional study with duodenal-jejunal exclusion. Obes Surg 2009;19:1077-83.

[27] Strader AD. Ileal transposition provides insight into the effectiveness of gastric bypass surgery. Physiol Behav 2006; 88: 277-82.

[28] DePaula AL, Macedo AL, SchraibmanV, Mota BR Vencio S. Hormonal evaluation following laparoscopic treatment of type 2 diabetes mellitus patients with BMI 20-34. Surg Endosc 2009;23:1724-32.

[29] DePaula AL, Macedo AL, Mota BR, Schraibman V. Laparoscopic ileal interposition associated to a diverted sleeve gastrectomy is an effective operation for the treatment of type 2 diabetes mellitus patients with BMI 21-29. Surg Endosc 2009;23:1313-20.

[30] Thaler JR Cummings DE. Minireview: Hormonal and metabolic mechanisms of diabetes remission after gastrointestinal surgery. Endocrinology 2009; 150:2518-25.

[31] Ferrannini E, Mingrone G. Impact of different bariatric surgical procedures on insulin action and beta-cell function in type 2 diabetes. Diabetes Care 2009;32:514-20.

[32] Letiexhe MR, Scheen AJ, Gérard PL, Desaive C, Lefèbvre PJ. Post-gastroplasty recovery of ideal body weight normalizes glucose and insulin metabolism in obese women. J Clin Endocrinol Metab 1995;80:364-9. 
Published in : Diabetes \& Metabolism (2009), vol. 35

Status : Postprint (Author's version)

[33] Letiexhe MR, Desaive C, Lefèbvre PJ, Scheen AJ. Intact cross-talk between insulin secretion and insulin action after post-gastroplasty recovery of ideal body weight in severely obese patients. Int J Obesity 2004;28:821-3.

[34] Kadera BE, Lum K, Grant J, Pryor AD, Portenier DD, DeMaria EJ. Remission of type 2 diabetes after Roux-en-Y gastric bypass is associated with greater weight loss. Surg Obes Relat Dis 2009;5:305-9.

[35] Lin E. Davis SS, Srinivasan J, Sweeney JF, Ziegler TR, Phillips L, Gletsu-Miller N. Dual mechanism for type-2 diabetes resolution after Roux-en-Y gastric bypass. Am Surg 2009;75:498-502.

[36] Cummings DE. Endocrine mechanisms mediating remission of diabetes after gastric bypass surgery. Int J Obes 2009;33(Suppl 1):S3340 .

[37] Knop FK. Resolution of type 2 diabetes following gastric bypass surgery: involvement of gut-derived glucagon and glucagonotropic signalling? Diabetologia 2009;52:2270-6

[38] Buchwald H, Estok R, Fahrbach H, Banel D, Jensen MD, Pories WJ, et al. Weight and type 2 diabetes after bariatric surgery: systematic review and meta-analysis. Am J Med 2009;122:248-56.

[39] Luyckx FH, Scheen AJ, Desaive C et al. Effects of gastroplasty on body weight and related biological abnormalities in morbid obesity. Diabetes Metab 1998;24:355-61.

[40] Sjöström L, Lindroos A-K, Peltonen M, Torgerson J, Bouchard C, Carlsson B, et al for the Swedish Obese Subjects Study Scientific Group. Lifestyle, diabetes, and cardiovascular risk factors 10 years after bariatric surgery. N Engl J Med 2004;351:2683-93.

[41] Sjöström L, Narbro K, Sjöström CD, KarasonK, Larsson B, Wedel H, et al. Effects of bariatric surgery on mortality in Swedish obese subjects. N Engl J Med 2007;357:741-52.

[42] Adams TD, Gress RE, Smith SC, Halverson RC, Simper SC, Rosamond WD, et al. Long-term mortality after gastric bypass surgery. N Engl J Med 2007;357:753-61

[43] MacDonald KG Jr, Long SD, Swanson MS, Brown BM, Morris P. Dohm GL, Pories WJ. The gastric bypass operation reduces the progression and mortality on non-insulin-dependent diabetes mellitus. J Gastrointest Surg 1997;1:213-20.

[44] Picot J, Jones J, Colquitt J, Gospodarevskaya E, Loveman E, Baxter L, Clegg A. The clinical effectiveness and cost-effectiveness of bariatric (weight loss) surgery for obesity: a systematic review and economic evaluation. Health Technol Assess 2009;13:1-358.

[45] Chevallier JM, Paita M, Rodde-Dunet MH, Marty M, Nogues F, Slim K, Basdevant A. Predictive factors of outcome after gastric banding: a nationwide survey on the role of center activity and patients'behavior. Ann Surg 2007;246:1034-9.

[46] Purnell JQ, Flum DR. Bariatric surgery and diabetes: who should be offered the option of remission? JAMA 2009;301:1593-5.

[47] Buchwald H, Estok R, Fahrbach K, Banel D, Sledge I. Trends in mortality in bariatric surgery: a systematic review and meta-analysis. Surgery 2007;142:621-35.

[48] Spanakis E, Gragnoli C. Bariatric surgery, safety and type 2 diabetes. Obes Surg 2009;18:363-8.

[49] Buchwald H, Consensus Conference Panel 2005 Consensus Conference Panel. Bariatric surgery for morbid obesity: health implications for patients, health professionals, and third-party payers. J Am Coll Surg2005;200:593-604

[50] Fried M, Hainer V, Basdevant A, Buchwald H, Deitel M, Finer N, et al. Interdisciplinary European guidelines for surgery for severe (morbid) obesity. Obes Surg 2007;17:260-70.

[51] Rubino F, Gagner M. Potential of surgery for curing type 2 diabetes mellitus. Ann Surg 2002;236:554-9. 